\title{
CONVEX SETS WITH THE LIPSCHITZ FIXED POINT PROPERTY ARE COMPACT
}

\author{
P. K. LIN ${ }^{1}$ AND Y. STERNFELD
}

\begin{abstract}
Let $K$ be a noncompact convex subset of a normed space $X$. It is shown that if $K$ is not totally-bounded then there exists a Lipschitz self map $f$ of $K$ with $\inf \{\|x-f(x)\|: x \in K\}>0$, while if $K$ is totally-bounded then such a map does not exist, but still $K$ lacks the fixed point property for Lipschitz mappings. It follows that a closed convex set in a normed space has the fixed point property for Lipschitz maps if and only if it is compact.
\end{abstract}

1. Introduction. In [4] Klee proves that a noncompact convex set in a normed space lacks the fixed point property for continuous maps.

In this note we extend this result to Lipschitz mappings.

Let $(K, d)$ and $(S, \rho)$ be metric spaces. A function $f: K \rightarrow S$ is a Lipschitz map if

$$
\|f\|_{L}=\sup \left\{\frac{\rho(f(x), f(z))}{d(x, z)}: x, z \in K\right\}<\infty .
$$

$(K, d)$ has the Lipschitz fixed point property (L.f.p.p.) if every Lipschitz self map of $K$ has a fixed point. $(K, d)$ is said to have the approximate Lipschitz fixed point property (approx. L.f.p.p.) if for every Lipschitz self map $f$ of $K \inf \{d(x, f(x))$ : $x \in K\}=0$. Our main result is the following

THEOREM 1. Let $K$ be a noncompact convex set in a normed space.

(i) If $K$ is not totally-bounded then it lacks the approx. L.f.p.p.

(ii) If $K$ is totally-bounded then it has the approx. L.f.p.p., but lacks the L.f.p.p.

In [2] Benyamini and the second named author show that the closed unit ball in an infinite dimensional normed space lacks the approx. L.f.p.p. Applying Theorem 1(i) to Banach spaces we obtain a more general result.

THEOREM 2. A closed noncompact convex set in a Banach space lacks the approx. L.f.p.p.

Combining Theorem 1 with the Schauder fixed point theorem we obtain

Theorem 3. A convex set in a normed space has the L.f.p.p. if and only if it is compact.

Received by the editors June 2, 1984.

1980 Mathematics Subject Classification. Primary 47H10.

${ }^{1}$ Partially supported by NSF grant DMS- 8201635 . 
In $\S 2$ we introduce a metric space $\Delta$ which plays a central role in the proof of Theorem 1, and state 3 propositions which expose the main properties of $\Delta$. Then we prove Theorem 1. The propositions will be proved in subsequent sections. Through this note the word "space" will always refer to a metric space, and "function" or "map" to Lipschitz mappings, even if not stated explicitly.

2. The space $\Delta$ and proof of Theorem 1. For a set $X$ let $l_{\infty}(X)$ denote the Banach space of bounded real valued functions on $X$ with the norm $\|f\|_{\infty}=\sup \{|f(x)|$ : $x \in X\}$. In particular for the set $N$ of positive integers $l_{\infty}=l_{\infty}(N)$ is the Banach space of bounded real sequences $x=\left(x_{1}, x_{2}, x_{3}, \ldots\right)$ with the norm $\|x\|_{\infty}=\sup _{n}\left|x_{n}\right|$. For $n \in N$ let $e_{n}=(0,0, \ldots, 0,1,0,0, \ldots) \in l_{\infty}$ be the $n$th unit vector, and set

$$
\Delta_{n}=\operatorname{conv}\left\{0, e_{n}, e_{n+1}\right\}, \quad n \in N ; \text { and } \Delta=\bigcup_{n \in N} \Delta_{n} .
$$

In subsequent sections we shall show that the metric space $\Delta$ (with the metric induced from $l_{\infty}$ ) enjoys the following properties:

Proposition 1. The space $\Delta$ as well as the spaces $R^{+}=\{t \in R: t \geqslant 0\}$ and $(0,1]=\{t \in R: 0<t \leqslant 1\}$ (with the metric induced from $R$ ) are Lipschitz absolute retracts.

DEFINITION. A space $X$ is a Lipschitz absolute retract (L.A.R.) if whenever a space $Y$ contains $X$ as a closed set, there exists a Lipschitz retraction $r: Y \rightarrow X$.

A mapping $h: K \rightarrow S$ is a Lipschitz equivalence if $h$ is Lipschitz, one-to-one, and $h^{-1}$ is Lipschitz. If there exists a Lipschitz equivalence of $K$ onto $S$ then $K$ and $S$ are said to be Lipschitz equivalent.

Two metric functions $d$ and $\rho$ on a set $K$ are equivalent if the identity map id: $(K, d) \rightarrow(K, \rho)$ is a Lipschitz equivalence.

Proposition 2. Let $K$ be a noncompact convex subset of a normed space.

(i) If $K$ is not totally-bounded then it contains a closed set which is Lipschitz equivalent to either $\Delta$ or $R^{+}$.

More precisely: If some bounded subset of $K$ is not totally-bounded then $K$ contains a closed set which is Lipschitz equivalent to $\Delta ;$ while if some ball $\left\{x \in K:\left\|x-x_{0}\right\| \leqslant 1\right\}$ in $K$ is totally-bounded (and $K$ itself is not) then $K$ contains a closed set which is Lipschitz equivalent to $R^{+}$.

(ii) If $K$ is totally-bounded then it contains a closed set which is Lipschitz equivalent to $(0,1]$.

Proposition 3. $\Delta$ lacks the approx. L.f.p.p.

Proof of Theorem 1. Theorem 1(i), and the second part of Theorem 1(ii) follow from Propositions 1, 2, and 3, and the following

Lemma. Let $(A, d)$ be a metric space, and let $B$ be a Lipschitz retract of $A$. If $B$ lacks the L.f.p.p. (the approx. L.f.p.p.) then so does $A$.

Proof. We prove for the approx. L.f.p.p. Let $r: A \rightarrow B$ be a retraction, and let $f$ : $B \rightarrow B$ be a map with $\inf \{d(x, f(x)): x \in B\}=\alpha>0$. Let $g: A \rightarrow A$ be defined by 
$g=f \circ r$, and $\varepsilon=\alpha /\left(\|g\|_{L}+2\right)$. (Note that $\|g\|_{L} \leqslant\|f\|_{L} \cdot\|r\|_{L}$.) Let $a \in A \backslash B$. If $d(a, B) \geqslant \varepsilon$ then $d(a, g(a)) \geqslant d(a, B) \geqslant \varepsilon$. If $d(a, B)<\varepsilon$ let $b \in B$ be such that $d(a, b)<\varepsilon$, and then

$$
d(a, g(a)) \geqslant d(b, g(b))-d(a, b)-d(g(a), g(b)) \geqslant \alpha-\varepsilon-\|g\|_{L} \varepsilon=\varepsilon .
$$

Hence $d(a, g(a)) \geqslant \varepsilon$ for all $a \in A$.

For the first part of Theorem 1(ii), let $K$ be a noncompact totally-bounded convex subset of a normed space $X$, and let $f: K \rightarrow K$ be a Lipschitz map. Let $\tilde{X}$ denote the completion of $X$, and $\bar{K}$ the closure of $K$ in $\tilde{X}$. Then $\bar{K}$ is compact, and $f$ admits an extension $\tilde{f}: \bar{K} \rightarrow \bar{K}$. By the Schauder theorem $\tilde{f}$ has a fixed point $x_{0} \in \bar{K}$. Let $\left\{x_{n}\right\}_{n \in N} \subset K$ be a sequence which converges to $x_{0}$. Then $\left\{x_{n}\right\}$ is an approximate fixed point for $f$, i.e. $\lim _{n} d\left(x_{n}, f\left(x_{n}\right)\right)=0$, and it follows that $K$ has the approx. L.f.p.p.

\section{L.A.R.'s, L.A.E.'s, and proof of Proposition 1.}

(1) Definition. A metric space $X$ is a Lipschitz absolute extensor (L.A.E.) if for every space $W$, a closed subset $Z$ of $W$, and a map $f: Z \rightarrow X, f$ admits an extension $\tilde{f}: W \rightarrow X$. If there exists a $\lambda \geqslant 1$ such that $\|\tilde{f}\|_{L} \leqslant \lambda\|f\|_{L}$, then $X$ is said to be a $\lambda$ L.A.E.

(2) ExAmple [6, Theorem 1]. $R$ is a 1 L.A.E.

Proof. Let $f: Z \rightarrow R$ be a map, then $\tilde{f}(w)=\sup \left\{f(z)-\|f\|_{L} d(z, w): z \in Z\right\}$ is an extension of $f$ with $\|\tilde{f}\|_{L}=\|f\|_{L}$.

(3) Corollary. For every set $A, l_{\infty}(A)$ is a 1 L.A.E.

Proof. Apply (2) to each coordinate $f(a, \cdot), a \in A$, of a map $f: Z \rightarrow l_{x}(A)$.

(4) TheOREM. A metric space $X$ is a L.A.R. if and only if it is a L.A.E.

The following Lipschitz version of a theorem by Hausdorff [3] will be applied in the proof of Theorem 4. Our proof follows that of Arens [1]. (See also [5] for a local version.)

(5) Theorem. Let $X$ and $W$ be spaces, $Z \subset W$ closed, and $f: Z \rightarrow X$ a map. There exists a space $Y$ which contains $X$ (isometrically) as a closed set, and an extension $g$ : $W \rightarrow Y$ of $f$.

Proof. Note first that $X$ is isometric to a subset of $l_{\infty}(X) .\left(x \rightarrow d(x, \cdot)-d\left(\cdot, x_{0}\right)\right.$ is an isometry, where $x_{0} \in X$ is some fixed point.) Set $B=l_{\infty}(X) \times R$, we realize $l_{\infty}(X)$ in $B$ as $l_{\infty}(X) \times\{0\}$, and we may assume that $X \subset l_{\infty}(X) \times\{0\} \subset B$.

So, in particular $f: Z \rightarrow l_{\infty}(X) \times\{0\}$, and since this is a L.A.E. (by Corollary (3)) $f$ admits an extension $h: W \rightarrow l_{\infty}(X) \times\{0\}$. Let $g: W \rightarrow B$ be defined by $g(w)=$ $h(w)+(0, d(w, Z))$ and set $Y=g(W) \cup X$. One checks easily that $X$ is closed in $Y$ and that $g \mid Z=f$.

Proof of Theorem 4. L.A.E. $\Rightarrow$ L.A.R. Let $X$ be a L.A.E., and let $Y$ contain $X$ as a closed set. Then an extension $r: Y \rightarrow X$ of the identity mapping id: $X \rightarrow X$ is a retraction.

L.A.R. $\Rightarrow$ L.A.E. Let $X$ be a L.A.R., let $Z \subset W$ be closed, and let $f: Z \rightarrow X$ be a map. By (5), there exists a space $Y$ which contains $X$ as a closed set, and an 
extension $g: W \rightarrow Y$ of $f$. Since $X$ is a L.A.R. there exists a retraction $r: Y \rightarrow X$. Then $\tilde{f}=r \circ g: W \rightarrow X$ is an extension of $f$.

(6) Corollary. A retract of a L.A.R. is a L.A.R.

Proof. Let $X$ be a retract of a L.A.R. $Y$ with a retraction $r: Y \rightarrow X$. We prove the $X$ is a L.A.E. Let $Z \subset W$ be closed, and $f: Z \rightarrow X$ be given. Then also $f: Z \rightarrow Y$, and since $Y$ is a L.A.E. there exists an extension $g: W \rightarrow Y$ of $f$. It follows that $\tilde{f}=r \circ g: W \rightarrow X$ is an extension of $f$.

(7) Corollary. If $X$ is Lipschitz equivalent to a L.A.R. then it is a L.A.R.

Proof. This is trivial for a L.A.E., and hence follows from (4).

(8) Proposition. There exists a Lipschitz retraction $r: l_{\infty} \rightarrow \Delta$.

Proof. We consider $l_{\infty}$ as a lattice with the natural order. Note that for $x \in \Delta$ and $y \in l_{\infty} 0 \leqslant y \leqslant x$ implies $y \in \Delta$. Let $e=(1,1,1, \ldots) \in l_{\infty}$, and $x \in l_{\infty}$. Set

$$
E(x)=\{\varepsilon: \varepsilon \geqslant 0,(x-\varepsilon e) \wedge 0 \in \Delta\} .
$$

Clearly $\|x\| \in E(x)$. Let $\varepsilon: l_{\infty} \rightarrow R^{+}$be defined by $\varepsilon(x)=\inf E(x)$. Then $\varepsilon$ is a Lipschitz map with $\|\varepsilon\|_{L}=1$. Indeed, for $x$ and $y$ in $l_{\infty}, x \leqslant y+\|x-y\|$. Hence $\varepsilon(y)+\|x-y\| \in E(x)$ and it follows that $\varepsilon(x) \leqslant \varepsilon(y)+\|x-y\|$, and by symmetry $|\varepsilon(x)-\varepsilon(y)| \leqslant\|x-y\|$. Let now $r: l_{\infty} \rightarrow \Delta$ be defined by $r(x)=(x-\varepsilon(x) \cdot e) \wedge 0$. Then $r$ is a retraction and $\|r\|_{L} \leqslant 2$.

Proof of Proposition 1. The fact that $\Delta$ is a L.A.R. follows from (6), (3), and (8). Since $R^{+}$is a retract of $R$, it is a L.A.R., too. To prove that $(0,1]$ is a L.A.R., we show that $[0,1)$ is a L.A.E. So let $Z \subset W$ closed and $f: Z \rightarrow[0,1)$ be given. Then also $f: Z \rightarrow[0,1]$ and since $[0,1]$ is clearly a L.A.E., there exists an extension $g: W \rightarrow[0,1]$ of $f$. Then

$$
\tilde{f}(w)=g(w) \frac{1}{1+d(w, Z)}: W \rightarrow[0,1)
$$

is an extension of $f$.

4. Proof of Proposition 2. Let $K$ be a noncompact convex subset of a normed space $X$. We distinguish between the following two cases: Case (i): $K$ is not totally-bounded, and Case (ii): $K$ is totally-bounded.

Cases (i). Here also we separate the proof into two cases.

Cases (i)a. Some bounded subset of $K$ is not totally-bounded. In this case we may assume without loss of generality that $0 \in K$, and that $K_{1}=\{x \in K:\|x\| \leqslant 1\}$ is not totally-bounded. Hence, there exists some $r>0$ such that $K_{1}$ cannot be covered by finitely many balls of radius $2 r$. It follows that

1. For every finite-dimensional linear subspace $Y$ of $X$, there exists some $x \in K_{1}$ with $d(Y, x) \geqslant r$.

Indeed, if not then $Y+B_{r} \supset K_{1}$ (where $B_{r}=\{x \in X:\|x\| \leqslant r\}$ ) and from the compactness of $\{y \in Y:\|y\| \leqslant 2\}$ it follows that finitely many balls of radius $2 r$ cover $K_{1}$. 
Now we select inductively a sequence $\left\{x_{n}\right\}_{n \geqslant 1}$ in $K_{1}$ as follows: let $x_{1} \in K_{1}$ be any element with $\left\|x_{1}\right\| \geqslant r$. Assume that $x_{1}, x_{2}, \ldots, x_{n}$ have been selected. Set $Y=\operatorname{span}\left\{x_{i}\right\}_{i=1}^{n}$, and apply 1 to find $x_{n+1} \in K_{1}$ with $d\left(Y, x_{n+1}\right) \geqslant r$.

For $n \geqslant 1$ set

$$
\Delta_{n}^{\prime}=\operatorname{conv}\left\{0, x_{n}, x_{n+1}\right\} \quad \text { and } \quad \Delta^{\prime}=\bigcup_{n \geqslant 1} \Delta_{n}^{\prime} .
$$

Then $\Delta^{\prime}$ is a closed subset of $K$ and is Lipschitz equivalent to $\Delta$ by the map $h$ : $\Delta \rightarrow \Delta^{\prime}$ which is defined by $h(0)=0, h\left(e_{n}\right)=x_{n}, n \geqslant 1$, and $h$ is linear on each $\Delta_{n}$. The verification of this fact is left to the reader.

Case (i)b. Some ball $\left\{x \in K:\left\|x-x_{0}\right\| \leqslant 1\right\}$ is totally-bounded. Note that in this case $K$ must be unbounded. Again we assume $0 \in K$. Let $\tilde{X}$ be the completion of $X$, and let $\bar{K}$ be the closure of $K$ in $\tilde{X}$. Set $\bar{K}_{n}=\{x \in \bar{K}:\|x\| \leqslant n\}$. Then $\bar{K}_{1}$ is compact, and since $(n+1)^{-1} \bar{K}_{n+1} \subset n^{-1} \bar{K}_{n} \subset \bar{K}_{1}$, and $n^{-1} \bar{K}_{n}$ contains a unit vector for each $n, \bigcap_{n \geqslant 1} n^{-1} \bar{K}_{n}$ must contain some vector $y_{0}$ with $\left\|y_{0}\right\|=1$. Then $t y_{0} \in \bar{K}$ for all $t \in R^{+}$. For each $n \geqslant 1$ pick some $x_{n} \in K$ with $\left\|n y_{0}-x_{n}\right\|<(n+10)^{-1}$. Then $\bigcup_{n \geqslant 1}\left[x_{n}, x_{n+1}\right]$ is a closed subset of $K$ which is Lipschitz equivalent to $R^{+}$.

Case (ii). Once again let $\bar{K}$ denote the closure of $K$ in the completion of $X$. Pick $x_{0} \in \bar{K} \backslash K$ and $x_{1} \in K$. For $n \geqslant 2$ select $x_{n} \in K$ such that

$$
\left\|\left(\left(1-\frac{1}{n}\right) x_{0}+\frac{1}{n} x_{1}\right)-x_{n}\right\|<2^{-(n+10)} .
$$

Then $\bigcup_{n \geqslant 1}\left[x_{n}, x_{n+1}\right]$ is a closed subset of $K$ which is Lipschitz equivalent to $(0,1]$.

5. A fixed point free map on $\Delta$. In this section we construct a Lipschitz map $f$ : $\Delta \rightarrow \Delta$ without an approximate fixed point, i.e. $\inf \{\|x-f(x)\|: x \in \Delta\}>0$.

For $x=\left(x_{1}, x_{2}, \ldots\right) \in \Delta$, let $\left\|x_{1}\right\|=\sum_{i=1}^{\infty}\left|x_{i}\right|$ denote the $l_{1}$ norm of $x$. Note that the metric functions induced on $\Delta$ by $\|x\|_{1}$ and $\|x\|_{\infty}=\sup _{i}\left|x_{i}\right|$ are Lipschitz equivalent, and we apply the $l_{1}$ norm in the construction of $f$.

First we define $f$ on $\frac{1}{3} \Delta=\left\{x \in \Delta:\|x\|_{1} \leqslant \frac{1}{3}\right\}$ as follows:

$f(0)=e_{1}, f\left(\frac{1}{3} e_{1}\right)=e_{1}, f\left(\frac{1}{3} e_{n}\right)=0$ for $n \geqslant 2$, and $f$ is linear on $\frac{1}{3} \Delta_{n}$ for each $n \geqslant 1$. Note that $f\left(\frac{1}{3} \Delta\right)=\left[0, e_{1}\right]$, and that for $x \in \frac{1}{3} \Delta_{n}, n \geqslant 2$, with $\|x\|_{1}=\frac{1}{3} f(x)=0$. As a piecewise linear map, $f$ is Lipschitzian on $\frac{1}{3} \Delta$.

Next, we define $f$ on $\left\{x \in \Delta:\|x\|_{1} \geqslant \frac{2}{3}\right\} ; f$ will map this set onto $\{x \in \Delta$ : $\left.\|x\|_{1}=1\right\}$. Let $g:\left\{x \in \Delta:\|x\|_{1}=1\right\} \rightarrow[1, \infty)=\{r \in R: r \geqslant 1\}$ be defined by

$$
\left.g\left((1-t) e_{n}+t e_{n+1}\right)\right)=n+t, \quad n \geqslant 1,0 \leqslant t<1 .
$$

$g$ is one-to-one and onto. We shall construct a map $h:\left\{x \in \Delta:\|x\|_{1} \geqslant \frac{2}{3}\right\} \rightarrow[1, \infty)$ and $f$ will be defined to be $f=g^{-1} \circ h$. $h$ is defined as follows:

For $x \in \Delta,\|x\|_{1}=\frac{2}{3}$,

$$
h(x)= \begin{cases}\frac{1}{2} g\left(\frac{x}{\|x\|_{1}}\right)+\frac{1}{2}, & x \in \Delta_{1}, \\ g\left(\frac{x}{\|x\|_{1}}\right)-\frac{1}{2}, & x \in \bigcup_{n \geqslant 2} \Delta_{n} .\end{cases}
$$



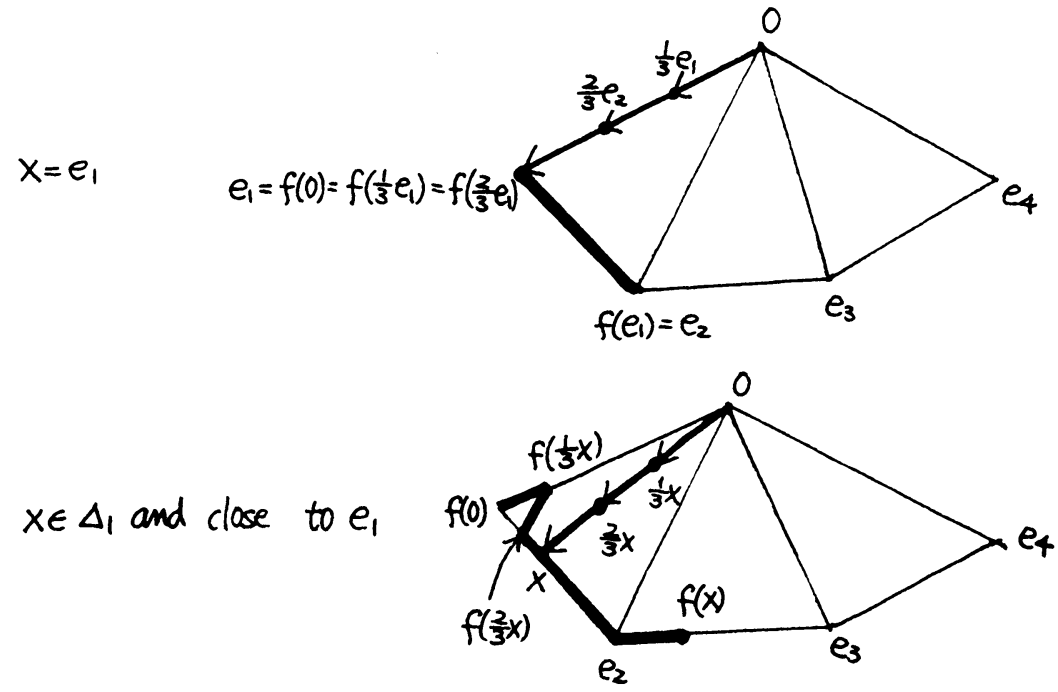

$x \in \Delta_{1}$ and close to $e_{2}$

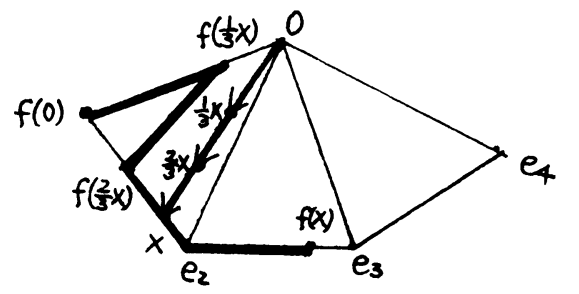

$$
x=e_{2}
$$

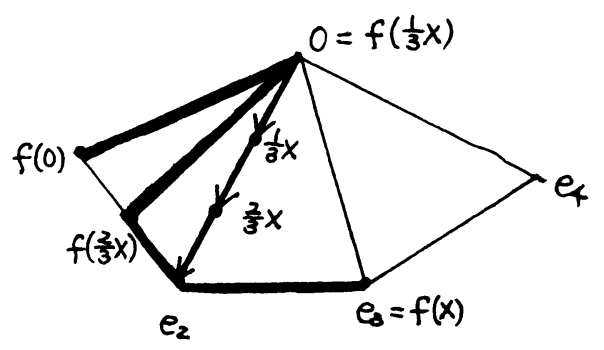

$x \in \Delta_{n} \quad n \geq 2$

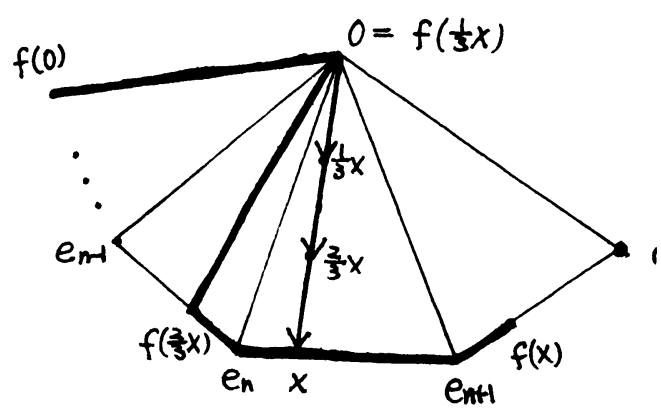

FIgURE 1 
And for $x \in \Delta,\|x\|_{1}=1, h(x)=g(x)+1$. For $x \in \Delta$ with $\frac{2}{3}<\|x\|_{1}, h$ is defined to be linear on $\left[\frac{2}{3}\left(x /\|x\|_{1}\right), x /\|x\|_{1}\right]$.

Under the metric $d(r, s)=|r-s| \wedge 1$ on $[0,1), g$ is a Lipschitz equivalence, and $h$ is Lipschitz. Hence $f=g^{-1} \circ h$ is Lipschitz.

To extend $f$ to the whole of $\Delta$, note that for $0 \neq x \in \Delta, f\left(\frac{1}{3}\left(x /\|x\|_{1}\right)\right)$ and $f\left(\frac{2}{3}\left(x /\|x\|_{1}\right)\right)$ are in the same $\Delta_{n}$ for some $n \geqslant 1$. Indeed, if $x \in \Delta_{1}$ then both are in $\Delta_{1}$, while if $x \notin \Delta_{1}$ then $f\left(\frac{1}{3}\left(x /\|x\|_{1}\right)\right)=0$. Hence we extend $f$ by letting it be linear on $\left[\frac{1}{3} x, \frac{2}{3} x\right]$ for all $x \in \Delta,\|x\|_{1}=1$. The extended map is still Lipschitzian. Figure 1 illustrates $[0, x]$ and $f([0, x])$ for several values of $x \in \Delta$ with $\|x\|_{1}=1$.

For $x \in \Delta$ with $\|x\|_{1}=1,[0, x] \cap f([0, x]) \subseteq\{0, x\}$, and since neither 0 nor $x$ (with $\|x\|_{1}=1$ ) are fixed points of $f, f$ is fixed point free. Hence, by compactness, $\inf \left\{\|x-f(x)\|: x \in \Delta_{1} \cup \Delta_{2}\right\}=\alpha>0$. But for $x \in \Delta_{n}, n>2$. $\|x-f(x)\|=$ $\|\tau x-f(\tau x)\|$, where $\tau: \Delta_{n} \rightarrow \Delta_{2}$ is the natural isometry. It follows that $\|x-f(x)\|$ $\geqslant \alpha$ for all $x \in \Delta$.

\section{REFERENCES}

1. R. Arens, Extension of functions on fully normal spaces, Pacific J. Math. 2 (1952), 11-22.

2. Y. Benyamini and Y. Sternfeld, Spheres in infinite dimensional normed spaces are Lipschitz contractible, Proc. Amer. Math. Soc. 88 (1983), 439-445.

3. F. Hausdorff, Erweiterung einer stetigen Abbildung, Fund. Math. 30 (1938), 40-47.

4. V. Klee, Some topological properties of convex sets, Trans. Amer. Math. Soc. 78 (1955), 30-45.

5. J. Luukkainen, Extension of spaces, maps and metrics in Lipschitz topologv, Ann. Acad. Sci. Fenn. Ser. A 17 (1978).

6. E. J. McShane, Extension of ranges of functions, Bull. Amer. Math. Soc. 40 (1934), 837-842.

Department of Mathematics, The University of Texas at Austin, Austin, Texas 78712

Department of Mathematics, University of Southern California, los Angeles, California 90007

Department of Mathematics, The University of Haifa, Haifa, Israel.

Current address (P. K. Lin): Department of Mathematics, University of Iowa, Iowa City, Iowa 52242 